\title{
An optical study of $X$-ray sources in the old open clusters NGC 752 and NGC 6940*
}

\author{
M. van den Berg and F. Verbunt
}

\author{
Astronomical Institute, Utrecht University, PO Box 80000, 3508 TA Utrecht, The Netherlands \\ Received 20 April 2001 / Accepted 22 May 2001
}

\begin{abstract}
We observed the optical counterparts of X-ray sources in the old open clusters NGC 752 and NGC 6940 to search for the origin of the X-rays. The photometric variability reported earlier for the blue straggler H 209 is not confirmed by our light curves, nor is an indication for variability seen in the spectra; thus its X-rays remain unexplained. The X-rays of VR 111 and VR 114 are likely not a result of magnetic activity as these stars lack strong Ca II H\&K emission, while in VR 108 the level of activity could be enhanced. The short-period binary H 313 is a photometric variable; this supports the interpretation that it is a magnetically active binary. From the detection of the Li I $6707.8 \AA$ line, we classify the giant in VR 84 as a first-ascent giant; this leaves its circular orbit unexplained. As a side-result we report the detection of Li I $6707.8 \AA$ in the spectrum of the giant H 3 and the absence of this line in the spectrum of the giant $\mathrm{H} 11$; this classifies $\mathrm{H} 3$ as a first-ascent giant and $\mathrm{H} 11$ as a core-helium-burning clump star, and confirms the faint extension of the red-giant clump in NGC 752.
\end{abstract}

Key words. stars: activity - stars: blue stragglers - stars: variables: general - open clusters and associations: individual: NGC 752, NGC 6940 - X-rays: stars

\section{Introduction}

X-ray observations of open clusters older than 1 Gyr led to the detection of many magnetically active binaries (e.g. Belloni 1997). This is not unexpected: at these high ages single late-type stars are believed to rotate too slowly to emit detectable X-rays, and tidal interaction in a close binary is therefore necessary to maintain rotation at a higher rate than is typical for stars of that age. However, our interest is mainly in the stars whose X-rays are not as easily explained: binaries with orbital periods too long for tidal interaction, and stars whose evolutionary statuses are poorly understood. Such peculiar X-ray sources were pointed out in M67 (Belloni et al. 1998) and were selected by us for an optical follow-up study (van den Berg et al. 1999). This revealed interesting results (e.g. the blue straggler S 1082 turned out to be a complex triple system, van den Berg et al. 2001a) but in several cases the $\mathrm{X}$-rays remain unexplained. The failure to explain the origin and properties of these stars demonstrates that our understanding of the X-rays and stellar interactions in old open clusters is still limited. We have observed similar

Send offprint requests to: M. van den Berg,

e-mail: m.c.vandenberg@astro.uu.nl

* Based on observations made with the Jacobus Kapteyn Telescope and the William Herschel Telescope operated on the island of La Palma by the Isaac Newton Group in the Spanish Observatorio del Roque de los Muchachos of the Instituto de Astrofisica de Canarias. peculiar X-ray sources in two other old clusters, NGC 752 and NGC 6940, to look for optical signatures for the X-ray emission and to compare their properties with those of the ones found in M 67. The properties of the X-ray sources in the two clusters are summarised in Table 1.

NGC 752 is a nearby ( $\sim 50$ pc, Daniel et al. 1994) open cluster with an estimated age of 2 Gyr (Dinescu et al. 1995). Seven X-ray sources were identified with optical cluster members (Belloni \& Verbunt 1996; not counting the probable detection of the short-period binary DS And). Our study is focused on the blue straggler $\mathrm{H} 209$, the only X-ray source in NGC 752 that is not located along the cluster's main sequence (see Fig. 1). H 209 is a spectroscopic binary with a long orbital period (Latham et al. private communication). Magnetic activity in the early-type star is not expected, but also the orbital period is too long to generate magnetic activity in a possible late-type secondary. Low-amplitude (0.05 mag) photometric variability was noted by Hrivnak (1977). Three of the remaining X-ray sources (H 205, H 313 and the contact binary H 235) are binaries with orbital periods less than 2 days; hence their X-rays are likely a result of magnetic activity. The same could be the case for the rapid rotator H 214 (Daniel et al. 1994). The radial-velocity measurements of $\mathrm{H} 182$ by Daniel et al. (1994) show no indication of binarity while no measurements are made of $\mathrm{H} 156$.

NGC 6940 is somewhat younger than NGC 752. It is considered to be an old cluster (Janes \& Phelps 1994) 
Table 1. Properties of the stars in NGC 752 and NGC 6940 discussed in this paper. We list the star identification number (Heinemann 1926; Vasilevskis \& Rach 1957), $V$ magnitude and $B-V$ colour (Daniel et al. 1994; Larsson-Leander 1964), the orbital period $P_{\text {orb }}$ (see Belloni \& Verbunt 1996 for references on NGC 752; rot $=$ rapid rotator $v_{\text {rot }} \sin i>30 \mathrm{~km} \mathrm{~s}^{-1}$, Daniel et al. 1994; see Mermilliod \& Mayor 1989 for NGC 6940), the X-ray count rate in PSPC channels 41-240 corresponding to 0.4-2.4 keV (Belloni \& Verbunt 1996; Belloni \& Tagliaferri 1997), the spectral type from literature (Garrison 1972; Rebeirot 1970; Larsson-Leander 1964), the spectral type as derived from our low-resolution spectra, the flux in the Ca II H\&K lines $F_{C a}$, the heliocentric radial velocity $v_{\text {rad }}$ and projected rotational velocity $v_{\text {rot }} \sin i$.

\begin{tabular}{|c|c|c|c|c|c|c|c|c|c|}
\hline ID & $V$ & $B-V$ & $\begin{array}{l}P_{\text {orb }} \\
\text { (days) }\end{array}$ & $\begin{array}{l}\text { ctrate } \\
\left(\mathrm{s}^{-1}\right)\end{array}$ & $\begin{array}{l}\text { sp.type } \\
\text { (literature) }\end{array}$ & sp.type & $\begin{array}{l}F_{\mathrm{Ca}} \\
\left(\mathrm{erg} \mathrm{s}^{-1} \mathrm{~cm}^{-2}\right)\end{array}$ & $\begin{array}{l}v_{\mathrm{rad}} \\
\left(\mathrm{km} \mathrm{s}^{-1}\right)\end{array}$ & $\begin{array}{c}v_{\text {rot }} \sin i \\
\left(\mathrm{~km} \mathrm{~s}^{-1}\right)\end{array}$ \\
\hline \multicolumn{10}{|l|}{ NGC 752} \\
\hline H 156 & 13.77 & 0.87 & & $0.0036(6)$ & & G9-K0V & & & \\
\hline H 182 & 12.24 & 0.61 & & $0.0038(6)$ & & F5-7V & & & \\
\hline H 205 & 9.92 & 0.42 & 1.45 & $0.0045(6)$ & F3IV-Vp & & & & \\
\hline Н 209 & 9.74 & 0.061 & $1574^{a}$ & $0.0018(5)$ & B9.5V & & & $10(3)$ & \\
\hline Н 214 & 10.47 & 0.38 & rot & $0.0039(6)$ & F2IV & & & $6.1(4)$ & $64(5)$ \\
\hline H 235 & 11.47 & 0.48 & 0.4118 & $0.0020(4)$ & F5 & & & & \\
\hline Н 313 & 13.39 & 0.83 & 1.95 & $0.0062(7)$ & & & & & \\
\hline $\mathrm{H} 3^{b}$ & 9.57 & 1.00 & & $<0.0084$ & KOIII & gG4-G8 & $8.7(2.2) 10^{-14}$ & $4.7(2)$ & - \\
\hline $\mathrm{H} 11^{b}$ & 9.29 & 0.97 & & $<0.0014$ & & $\mathrm{gG}$ & & $5.2(2)$ & - \\
\hline \multicolumn{10}{|c|}{ NGC 6940} \\
\hline VR 84 & 10.91 & 0.83 & 54.2 & $0.0020(5)$ & F8 & F4-F6 & & $1.4(4)$ & $4-5$ \\
\hline VR 100 & 10.43 & 0.74 & 82.5 & $0.0011(4)$ & $\mathrm{F} 5^{c}$ & F4-F6 & & $-15.8(1)$ & $1-2$ \\
\hline VR 108 & 11.19 & 1.04 & & $0.0018(5)$ & gG5 & gG4-G8 & $1.3(0.3) 10^{-14}$ & 7.7(1) & $5-6$ \\
\hline VR 111 & 11.56 & 1.05 & 3595 & $0.0011(4)$ & gG5 & gG5-G9 & $5.8(1.5) 10^{-15}$ & $10.0(6)$ & $3-4$ \\
\hline VR 114 & 11.17 & 1.27 & & $0.0012(5)$ & gG8 & gG9-K0 & $2.4(0.6) 10^{-15}$ & $-9.1(7)$ & $1-2$ \\
\hline VR $92^{b}$ & 11.77 & 1.20 & 549.2 & $<0.0010$ & gG8 & gG5-G9 & $6.4(1.6) 10^{-15}$ & $8.1(4)$ & $2-3$ \\
\hline $\operatorname{VR} 152^{b}$ & 10.84 & 1.07 & & $<0.0005$ & gG8 & gG5-G9 & & $8.2(5)$ & $1-2$ \\
\hline
\end{tabular}

${ }^{a}$ Preliminary solution by Latham et al. (private communication).

${ }^{b}$ Comparison stars, $2 \sigma$-upper limit to the PSPC count rate determined from re-analysis of ROSAT data).

${ }^{c}$ Spectrum is composite (Bidelman suggests G8II+A7, see Walker 1958).

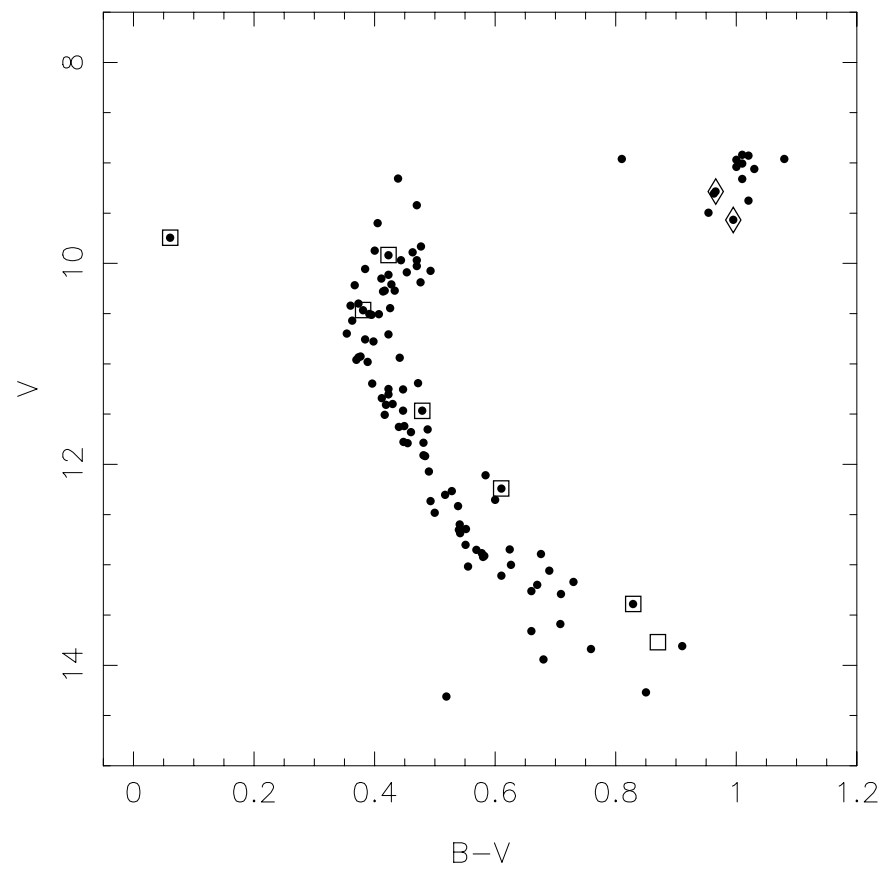

Fig. 1. Colour-magnitude diagram of NGC 752 showing the member stars according to Daniel et al. (1994). X-ray sources are indicated with squares, our comparison stars with diamonds. The empty square is at the position of $\mathrm{H} 156$ that is classified as a possible member by Daniel et al. with age-estimates ranging from 0.6 Gyr (Carraro \& Chiosi 1994) to 1.1 Gyr (van den Bergh \& McClure 1980). Four X-ray sources were identified with cluster members (Belloni \& Tagliaferri 1997, Fig. 2). VR 111 is a spectroscopic binary with a 3595-day eccentric orbit, too wide for tidal interaction (Verbunt \& Phinney 1995). For VR 108 the limit on the radial-velocity variations excludes orbital periods shorter than 4000 days (Mermilliod \& Mayor 1989). VR 114 is not a proper-motion member (Sanders 1972) and therefore not included in photometric and radial-velocity studies of the cluster. However, Belloni \& Tagliaferri derived that it is located at a similar distance as the cluster ( $\sim 900 \mathrm{pc}$, Larsson-Leander 1964) and suggested that the star could somehow be related to the cluster. We are mainly interested in these three stars. The other sources, VR 100 and VR 84, are spectroscopic binaries with circular orbits shorter than 100 days, indicative for tidal interaction. VR 100 is a double-lined spectroscopic binary which explains its position in the colour-magnitude diagram between the turnoff stars and the giants.

We have obtained low- and high-resolution spectra to look for optical signatures of X-ray activity. In particular we studied the Ca II H\&K lines that are optical indicators of magnetic activity, and we determined projected rotational velocities. For the X-ray sources in NGC 752 


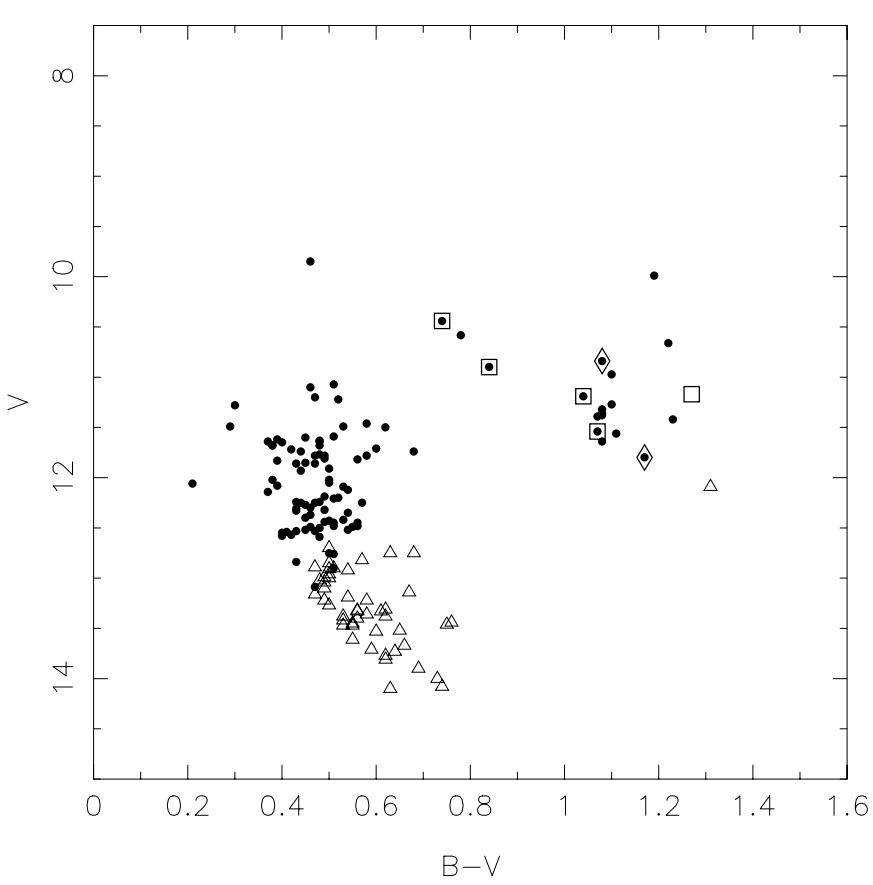

Fig. 2. Colour-magnitude diagram of NGC 6940 showing the stars with proper-motion membership probability larger than $70 \%$ (filled circles; Sanders 1972). No proper-motion information is available for the fainter stars, indicated with open triangles. X-ray sources are indicated with squares, our comparison stars with diamonds. The empty square is at the position of VR 114 that is not a proper-motion member according to Sanders (1972).

we also obtained multi-colour light curves. Section 2 describes our observations and data-analysis. The results are presented in Sect. 3 and are discussed in the last section.

\section{Observations and data reduction}

\subsection{Spectroscopy}

Low- and high-resolution spectra were obtained with the 4.2-m William Herschel Telescope on La Palma on the nights of July 29 and 30 1999. Weather conditions were good with a typical seeing of $1^{\prime \prime}$. An overview of the spectroscopic observations is given in Table 2 .

\subsubsection{Low-resolution spectra}

Low-resolution $(R \approx 1000)$ spectra were obtained with the double-beam spectrograph ISIS used in combination with the 5700-dichroic. The blue arm was centred on $4504 \AA$. The 300 lines $\mathrm{mm}^{-1}$ grating gives a dispersion of $\sim 0.86 \AA$ pixel $^{-1}$ which results in a wavelength coverage of 2743-6260 $\AA$. The atmosphere blocks flux below $3200 \AA$. The red arm was centred on $7191 \AA$ and was used with the 158 lines $\mathrm{mm}^{-1}$ grating. The dispersion of $\sim 2.90 \AA$ pixel $^{-1}$ results in a red spectrum of 5778-8726 $\AA$. Program stars were observed with a $4^{\prime \prime}$ slit width; the slit was widened to $8^{\prime \prime}$ for the observations of the flux standard $\mathrm{BD}+284211$.
Table 2. Log of the spectroscopic observations. From left to right: star number; date of observation: n(ight) $1=$ July 29, $2=$ July 30 1999; UT at start of the exposure and exposure time $t_{\exp }$ in seconds for ISIS (where appropriate, $t_{\exp }$ is listed separately for the blue/red arm), and the blue and red setup of UES.

\begin{tabular}{|c|c|c|c|c|c|c|c|}
\hline \multirow[t]{2}{*}{ ID } & \multirow{2}{*}{$\mathrm{n}$} & \multicolumn{2}{|c|}{ ISIS } & \multicolumn{2}{|c|}{ UES-b } & \multicolumn{2}{|c|}{ UES-r } \\
\hline & & UT & $t_{\exp }$ & UT & $t_{\exp }$ & UT & $t_{\exp }$ \\
\hline \multicolumn{8}{|c|}{ X-RAY SOURCES } \\
\hline Н 156 & 2 & $2: 43$ & 360 & & & & \\
\hline Н 182 & 2 & $2: 51$ & 120 & & & & \\
\hline \multirow[t]{9}{*}{ Н 209} & 1 & $3: 34$ & 20 & $4: 59$ & 720 & $4: 07$ & 360 \\
\hline & & & & & & $4: 19$ & 360 \\
\hline & & & & & & $5: 15$ & 720 \\
\hline & & & & & & $5: 30$ & 600 \\
\hline & & & & & & $5: 43$ & 300 \\
\hline & 2 & $2: 58$ & 20 & 4:09 & 720 & $3: 54$ & 720 \\
\hline & & & & & & $4: 47$ & 900 \\
\hline & & & & & & 5:05 & 900 \\
\hline & & & & & & $5: 44$ & 600 \\
\hline Н 214 & 2 & $2: 54$ & 30 & & & $5: 23$ & 600 \\
\hline \multirow[t]{2}{*}{ VR 84} & 2 & $0: 36$ & $90 / 40$ & $23: 30$ & 600 & $21: 41$ & 750 \\
\hline & & & & $23: 43$ & 600 & & \\
\hline \multirow[t]{2}{*}{ VR 100} & 1 & $2: 39$ & $30 / 15$ & $23: 20$ & 750 & $21: 11$ & 900 \\
\hline & & $2: 43$ & $60 / 30$ & & & & \\
\hline \multirow[t]{2}{*}{ VR 108} & 2 & $0: 33$ & 90 & $22: 54$ & 600 & $21: 14$ & 600 \\
\hline & & & & $23: 12$ & 600 & $21: 27$ & 600 \\
\hline \multirow[t]{2}{*}{ VR 111} & 1 & $2: 52$ & $180 /$ & $0: 19$ & 900 & $22: 02$ & 1200 \\
\hline & & & 120 & $0: 37$ & 900 & & \\
\hline \multirow[t]{2}{*}{ VR 114} & 1 & $2: 56$ & $180 / 90$ & $0: 55$ & 900 & $22: 26$ & 1200 \\
\hline & 2 & $0: 28$ & $180 / 90$ & $23: 57$ & 900 & & \\
\hline \multicolumn{8}{|c|}{ COMPARISON STARS } \\
\hline H 3 & 1 & $3: 37$ & $30 / 20$ & $4: 57$ & 500 & $4: 28$ & 600 \\
\hline H 11 & 2 & $2: 56$ & $20 / 10$ & $4: 26$ & 360 & $3: 44$ & 360 \\
\hline \multirow[t]{2}{*}{ VR 92} & 1 & $2: 48$ & 120 & $23: 44$ & 1000 & $21: 46$ & 1500 \\
\hline & & & & 0:06 & 1200 & & \\
\hline \multirow[t]{2}{*}{ VR 152} & 2 & $0: 39$ & $90 / 30$ & $21: 58$ & 600 & $22: 41$ & 750 \\
\hline & & $0: 40$ & $-/ 30$ & $22: 28$ & 600 & & \\
\hline \multicolumn{8}{|c|}{ RADIAL-VELOCITY STANDARDS } \\
\hline \multirow[t]{2}{*}{ HD $187691^{a}$} & 1 & & & $1: 27$ & 20 & $2: 12$ & 40 \\
\hline & 2 & & & $20: 51$ & 80 & $20: 57$ & 80 \\
\hline \multirow[t]{2}{*}{ HD $212943^{b}$} & 1 & & & $1: 41$ & 20 & $1: 57$ & 40 \\
\hline & 2 & & & 0:00 & 20 & $3: 29$ & 20 \\
\hline \multicolumn{8}{|c|}{ FLUX STANDARD } \\
\hline \multirow[t]{3}{*}{$\mathrm{BD}+284211$} & 1 & $3: 21$ & 30 & & & & \\
\hline & 2 & 1:01 & 30 & & & & \\
\hline & & $1: 03$ & $30 /-$ & & & & \\
\hline
\end{tabular}

${ }^{a} \mathrm{~F} 8 \mathrm{~V},{ }^{b} \mathrm{~K} 0 \mathrm{III}-\mathrm{IV}$ (Astronomical Almanac).

IRAF $^{1}$ routines were used to reduce the data. First a bias correction was applied to the frames. The dichroic leaves a signature in the blue spectra between 4000 and $4600 \AA$ that consists of wiggles with an amplitude of up

${ }^{1}$ IRAF is distributed by the National Optical Astronomy Observatories, which are operated by the Association for Universities routines. Inc., under cooperative agreement with the National Science Foundation. 
to $6 \%$ of the count level. This is not entirely removed by dividing the bias-corrected frames through a normalised flatfield image. Instead, the effect was reduced by extracting a spectrum from an unflatfielded image and next dividing it by a flatfield spectrum extracted from the same region on the CCD as the object spectrum.

Wavelength calibration was done with CuNe-lamp spectra. A dispersion solution was derived by fitting second (blue) and first (red) order cubic splines to the position of the arc lines on the CCD. Counts were converted to absolute flux using spectra of BD+28 4211 and the flux table by Massey et al. (1988) and the atmospheric extinction curve for La Palma (King 1985).

\subsubsection{High-resolution spectra}

The Utrecht Echelle Spectrograph (UES) was used in a blue (central wavelength $4253 \AA$ ) and red (central wavelength $6565 \AA)$ setup to obtain high-resolution $(R \approx$ $49000)$ spectra. The spectrograph was combined with the 31 lines $\mathrm{mm}^{-1}$ grating and SITe1 CCD, resulting in a total wavelength coverage of 3620 to $9930 \AA$ A. Exposures of a tungsten lamp served as flatfield images; thorium-argon lamp spectra were taken for the purpose of wavelength calibration.

The images were processed with the IRAF packages CCDRED and ECHELLE. They were corrected for a bias signal and flatfielded before spectra were extracted for each echelle-order. Sixth-order polynomials were fitted in both directions of the CCD to the positions of the ThArlines. The maximum residuals to the fit are $\sim 0.01 \AA$ (or $0.6 \mathrm{~km} \mathrm{~s}^{-1}$ at $5000 \AA$ ). Polynomials were fitted to the spectra for continuum normalisation. The orders containing the Ca II H\&K lines of a given star were calibrated in units of absolute flux with the calibrated low-resolution spectra of the same star.

\subsection{Photometry}

The X-ray sources in NGC 752 were monitored in the $U$, $B, V$ and $I$ bands during the seven nights of December 20 to 261999 with the 1-m Jacobus Kapteyn Telescope on La Palma. The typical seeing varied between $1^{\prime \prime} 6$ and $3^{\prime \prime}$. The telescope is equipped with a $2148 \times 2148$ pixel $^{2}$ SITe2 CCD which has an unvignetted field of view of $\sim 10 \times 10 \operatorname{arcmin}^{2}$. The field around the blue straggler H 209 was observed intensively with 4 to 5 exposures per filter per hour. Roughly every two $U B V I$-sequences the pointing was changed between a field that in addition includes either the X-ray source H 156 or H 182. The frames were exposed for typically $80 \mathrm{~s}$ in $U, 25 \mathrm{~s}$ in $B$ and $15 \mathrm{~s}$ in $V$ and $I$. Two fields including $\mathrm{H} 205, \mathrm{H} 214, \mathrm{H} 235$ and H 313 were observed only once or twice per night with typical exposure times of $180 \mathrm{~s}(U), 120 \mathrm{~s}(B), 60 \mathrm{~s}(V, I)$ for $\mathrm{H} 313$ and $80 \mathrm{~s}(U), 15 \mathrm{~s}(B), 10 \mathrm{~s}(V, I)$ for the other sources. The locations of the fields that were observed are shown in Fig. 3.
Bias removal and flatfielding were done in IRAF. Aperture photometry for all the stars in the fields was done with the DAOPHOT.PHOT task. The method of ensemble photometry (Honeycutt 1992) was used to compute differential light curves.

\section{Results}

\subsection{Photometric variability}

The light curves in each filter were tested for variability with a $\chi^{2}$-test. A star was labelled as a probable variable if the probability that its light curve is compatible with being constant is smaller than $0.3 \%$. Two stars were thus found to be variable: H313 and, as expected, the contact binary H 235. We computed Lomb-Scargle periodograms (Scargle 1982) to search for periodicity in the light curves of $\mathrm{H} 313$, that contain only $\sim 10$ points. A periodogram was computed at 1000 frequencies between a minimum period and maximum period that correspond to twice the sampling period ( 3 hours) and the length of the observation run ( 7 days). We chose the highest peak in the periodogram as a first estimate for the period of the variability. A false-alarm probability (fap) for the period detection was computed (van den Berg et al. 2001b) to estimate the chance detection of the periodicity. We accept periods with a fap less than $1 \%$ as significant, but the candidate periods all had higher faps. However, when the data points are folded on the binary period of 1.95 days, photometric variability on that period looks suggestive (Fig. 4). As an accurate photometric period of 0.4118 days is known for H 235 (Milone et al. 1995), we did not search for a period in its light curves of only $\sim 10$ points.

The light curves of our main target $\mathrm{H} 209$ are constant within $<0.01$ mag.

\subsection{Spectral classification}

With the classification criteria of Malyuto \& SchmidtKaler (1997) for F- to K-type stars, spectral types were assigned to $\mathrm{H} 156$ and $\mathrm{H} 182$, for which no classifications were found in literature. The method is based on features in the wavelength region 3640 to $5305 \AA$ that define the classification indices $I_{1}, I_{2}, I_{3}$ and $I_{S}$. We computed the values for these indices with the low-resolution spectra. A spectral class of G9-K0 V is derived for $\mathrm{H} 156$ and of F5-F7 for H 182; the values of the classification indices of H 182 are insensitive to luminosity class in that specific region, but its position in the colour-magnitude diagram shows it to be a dwarf. The comparison giant H 11 is classified as a G-type giant (luminosity class III or brighter). For the other stars we find spectral types that are in agreement with earlier classifications to within two subclasses (see Table 1). The spectral types of H 209 and H 214 are too early to be classified with the criteria of Malyuto \& Schmidt-Kaler. 


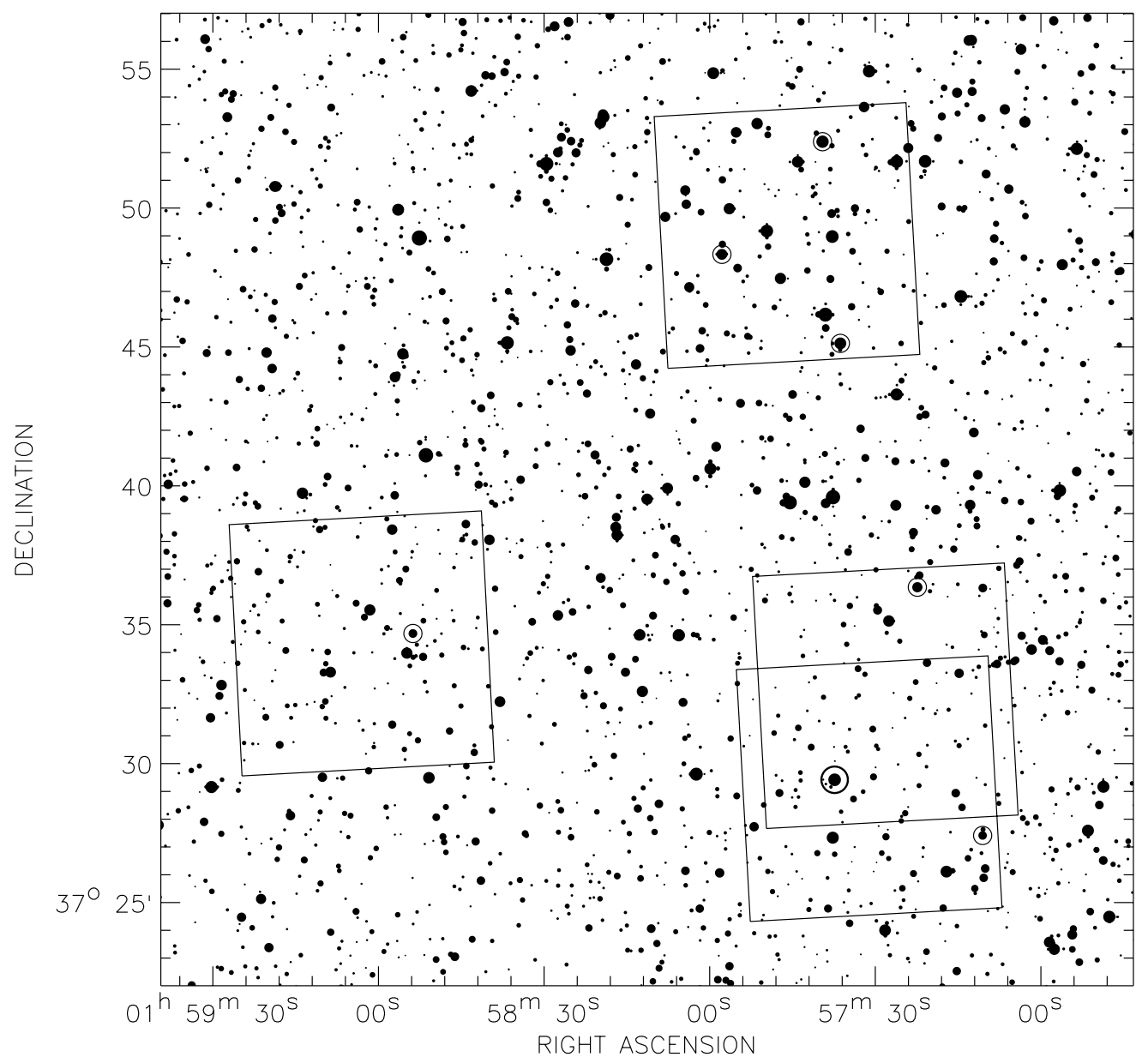

Fig. 3. Map of a $35 \times 35 \operatorname{arcmin}^{2}$ region of NGC 752 centred on $\alpha_{\mathrm{J} 2000}=1^{\mathrm{h}} 58^{\mathrm{m}} 11^{\mathrm{s}} .3, \delta_{\mathrm{J} 2000}=37^{\circ} 39^{\prime} 34^{\prime \prime}$. 0 . The fields that were monitored are indicated, X-ray sources are encircled. The coordinates of the stars in the field are taken from the USNO-A1.0 catalogue.

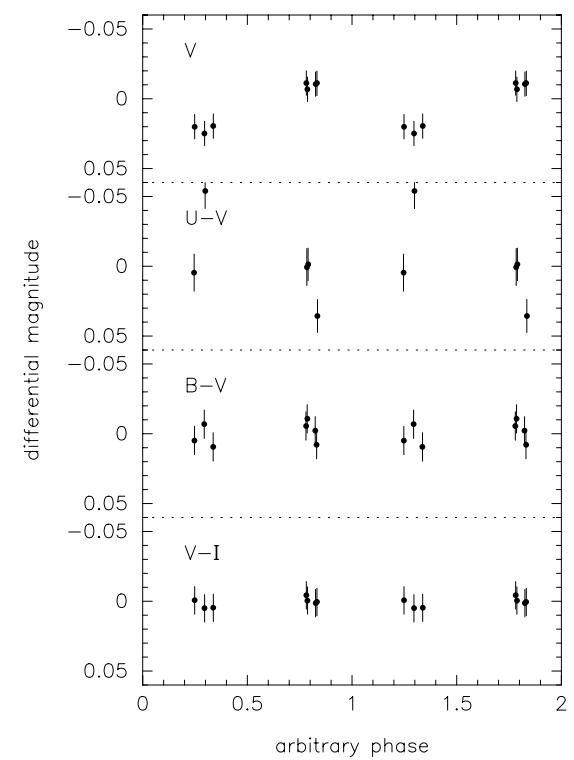

Fig. 4. Light and colour curves of $\mathrm{H} 313$ folded on the orbital period of 1.95 days. The magnitudes are plotted with respect to the average $V$-magnitude and colours.
With the low-resolution spectrum of VR 114 we derive its spectroscopic distance. The luminosity classification is refined using the calibration of the equivalent width of the Ca II infrared lines at 8498.02 and $8542.09 \AA$ against luminosity class by Mallik (1994). The equivalent width of $4.8 \AA$ (determined from the high-resolution spectrum between the ranges used by Mallik) indicates that VR 114 is probably of luminosity class II or III. $B-V$ $(1.24 \pm 0.14)$ and $U-B(1.08 \pm 0.14$, no measurement found in literature) colours are computed by folding the low-resolution spectrum calibrated in absolute-flux units with $U, B$ and $V$ filter transmission curves. Errors are estimated from comparison of the magnitudes thus derived for the flux standard with tabulated values. The reddening and distance can now be derived from comparison with the colours and absolute magnitudes of standard giant (class III) and bright giant (class II) stars. If VR 114 is a giant, then $E(B-V) \approx 0.3$ (for type K0) and the distance $\sim 810 \mathrm{pc}$, similar to the value found by Belloni \& Tagliaferri; if VR 114 is a bright giant, then $E(B-V) \approx 0.16$ (for type $\mathrm{K} 0$ ) and the distance $\sim 4 \mathrm{kpc}$. As the reddening in the direction of NGC 6940 was found 
to vary between 0.2 and 0.3 (Larsson-Leander 1964), the classification of VR 114 as a class III giant seems to be more plausible. We thus confirm that VR 114 lies at about the same distance as NGC 6940.

\subsection{Ca II H\&K emission}

Emission cores in the centre of the broad Ca II H\&K absorption lines are often used as optical indicators for magnetic activity in late-type stars (e.g. Fernández-Figueroa et al. 1994). Therefore, strong emission in these lines would be an indication that the X-rays are of coronal origin. The high-resolution spectra of our late-type target and comparison stars in the region around the $3933.67 \AA \mathrm{Ca}$ II K line are shown in Fig. 5. Emission above the level of the absorption profile is seen in all X-ray sources, but the emission is of similar strength as in the non-X-ray sources $\mathrm{H} 3$ and VR 92. The flux in the emission lines was estimated with the IRAF task SPLOT: the pseudo-continuum of the absorption line is estimated by eye; the flux above this level is obtained by summing the pixel values of the fluxcalibrated high-resolution spectra above a straight line connecting the continuum on both sides of the emission (see Table 1 ). We adopt an error of $\sim 25 \%$ accounting for the fact that the choice of the continuum is a bit arbitrary. We choose this method to measure emission fluxes as it is similar to the way fluxes were determined for the stars in our comparison sample (Fernández-Figueroa et al. 1994); higher-order fits to the continuum, as used by FernándezFigueroa et al., instead of a straight line to estimate the continuum gave similar results for the stars discussed in van den Berg et al. (1999). No emission flux is determined for VR 100, that has a composite spectrum, and for VR 84, whose spectrum could also be composite judging from its position in the colour-magnitude diagram. For VR 100 the predicted position of the K-line lies to the left of the centre in the composite absorption profile. Apparently, the relative contribution of the companion star is strong in the blue.

A comparison with magnetically active binaries is made by combining the information from the X-ray and $\mathrm{H} \& \mathrm{~K}$ emission. In Fig. 6 the emission in both activity indicators is plotted for the same sample of magnetically active binaries as used in van den Berg et al. (1999) to study magnetic activity of stars in M 67. The H\&K fluxes were taken from Fernández-Figueroa et al. (1994), the X-ray emission is given as the count rate in the 0.4 to $2.4 \mathrm{keV}$ band of the ROSAT PSPC. A description of the sample and the determination of the X-ray count rates can be found in van den Berg et al. (1999). The fluxes and count rates are multiplied by the square of the distance in parsecs so that stars at different distances can be compared. To correct for the absorption towards NGC 6940, we multiply the X-ray count rate and Ca II H\&K optical fluxes as listed in Table 1 by 3.8 and 3.3, respectively. Our target stars lie in the region where active binaries are found, but the level of activity in VR 111 and VR 114 appears to be low for their luminosity class. This makes it unlikely that

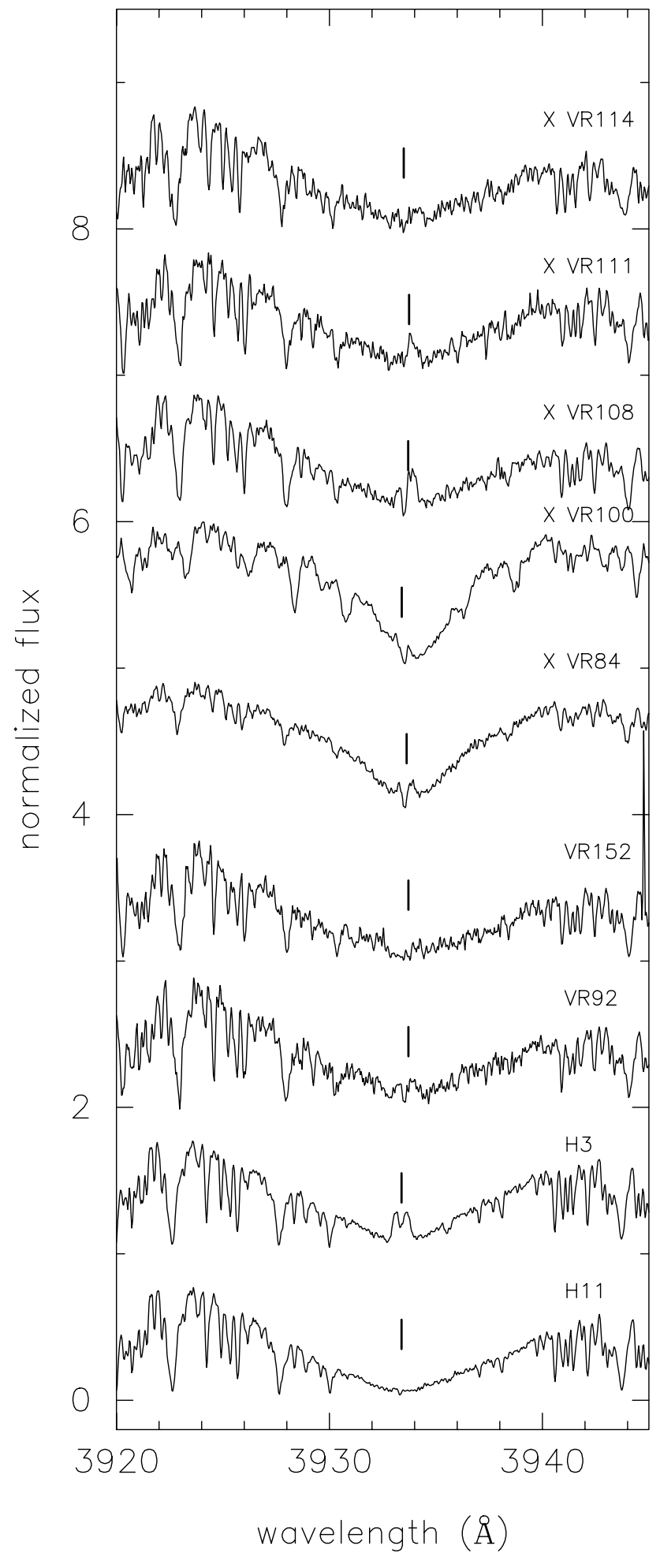

Fig. 5. Ca II K line in our late-type X-ray sources ("X") and the non-X-ray sources comparison giants H 3, H 11, VR 92 and VR 152. The expected position of the K-line centre as derived from the radial-velocity measurements of Sect. 3.4 is marked with the vertical line; from the position of this line to the left of the centre of the absorption profile it is clear that the spectrum of VR 100 is composite. The spectra are normalised to the continuum flux near 3915 and $4000 \AA$. A vertical offset is applied for clarity to all spectra but that of $\mathrm{H} 11$. 


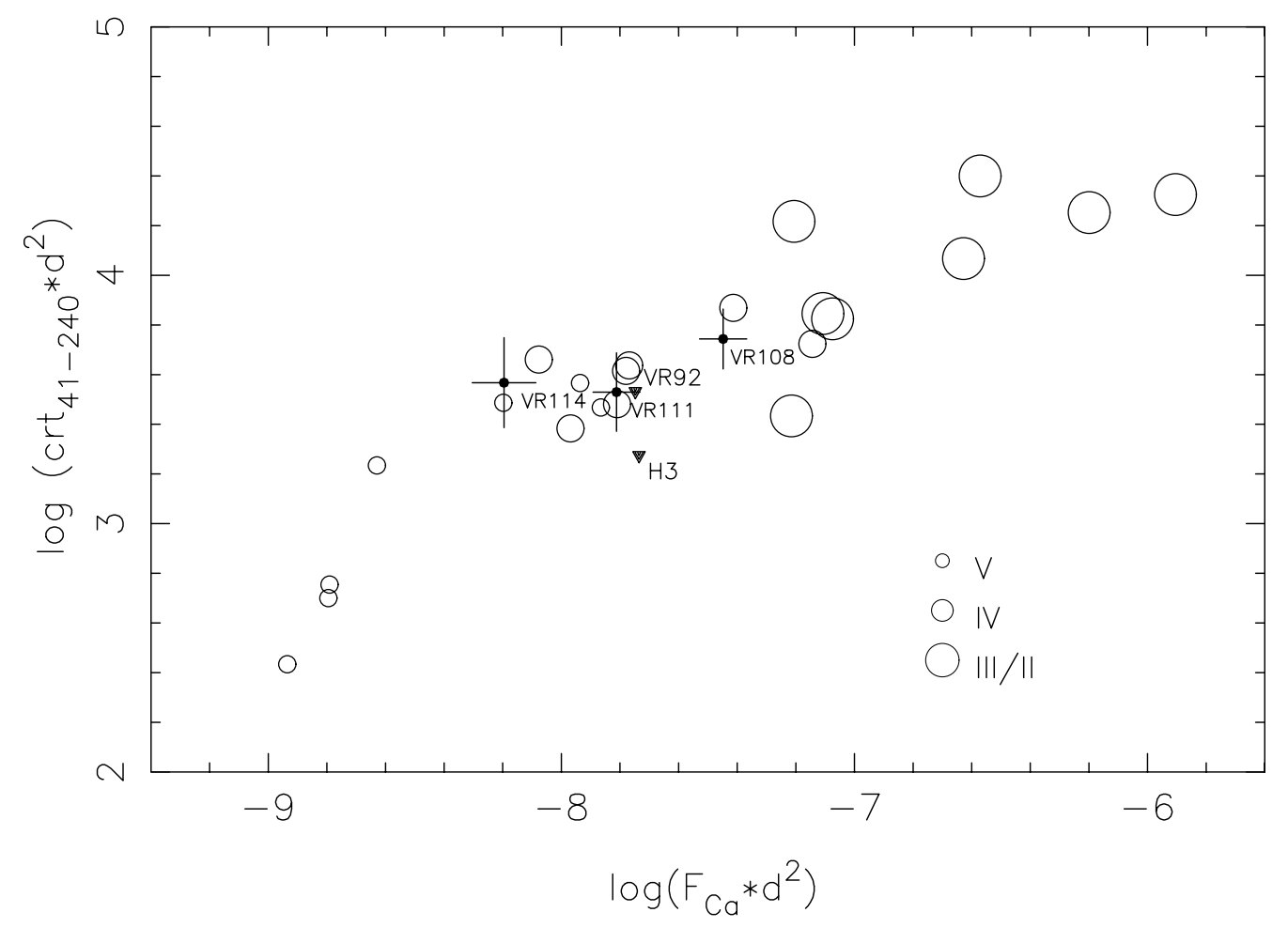

Fig. 6. Comparison of the Ca II H\&K and X-ray emission of our targets with that of magnetically active binaries. Fluxes in the Ca II H\&K emission lines $F_{\mathrm{Ca}}$ (in $\mathrm{erg} \mathrm{s}^{-1} \mathrm{~cm}^{-2}$ ) and X-ray count rates between 0.4 and $2.4 \mathrm{keV}$ (channel $41-240$ of the ROSAT PSPC) are multiplied with the square of the distance $d$ in parsec to eliminate the effect of distance. The luminosity class of the active star in the comparison sample is indicated with the size of the symbol. The comparison giants H 3 and VR 92 are indicated with filled triangles using the upper limits to the PSPC count rates listed in Table 1.

the X-ray emission arises through coronal activity in these giants. As the position of VR 108 lies closer to the active giants, activity could be enhanced in VR 108 .

No H\&K emission lines are visible in the low-resolution spectra of H 156, H 182 and H 214.

We also checked the X-ray sources for emission in the $\mathrm{H} \alpha$ line, another optical signature for activity, but found no indication for emission. The nine spectra of H 209 show no variability in the $\mathrm{H} \alpha$-profile.

\subsection{Radial and rotational velocities}

Radial velocities were determined through cross correlation of the high-resolution spectra against the spectra of the radial-velocity standards. We adopt the velocity resulting from correlation with the template that gives the highest correlation peak (better spectral match). The orders between 4960 and $5510 \AA$ for the blue exposures and 4970 and $5530 \AA$ for the red exposures were used; Table 1 lists their average. The systematic errors in the measurements for the rapid rotator $\mathrm{H} 214$ will be larger than in the other measurements as our radial-velocity standards are slow rotators and therefore give a bad match between object and template spectrum. The radial velocity of H 209 was measured by cross correlating the spectrum between 5145 and $5225 \AA$ with a synthetic spectrum (Kurucz 1979) of $T_{\text {eff }}=9750 \mathrm{~K}$, gravity $\log g=4.0$ and $v_{\text {rot }} \sin i=50 \mathrm{~km} \mathrm{~s}^{-1}$ (we adopt the parameters for $\mathrm{H} 209$ as derived by Schönberner \& Napiwotzki 1994).

The results for H3, H11, VR 152 and VR 108 agree with the values found by Mermilliod et al. (1998) and Mermilliod \& Mayor (1989) within $1 \mathrm{~km} \mathrm{~s}^{-1}$; the velocity we find for $\mathrm{H} 214$ is $2 \mathrm{~km} \mathrm{~s}^{-1}$ off from the value of Daniel et al. (1994). For the proper-motion non-member VR 114 we find a radial velocity that does not agree with the cluster mean $\left(7.75 \pm 0.13 \mathrm{~km} \mathrm{~s}^{-1}\right.$, Mermilliod \& Mayor 1989). Comparison with Fig. 5 of Mermilliod \& Mayor (1989) shows that the radial velocities of the binaries in NGC 6940 are roughly as expected, except for VR 84 for which we observe a radial velocity that is $\sim 7 \mathrm{~km} \mathrm{~s}^{-1}$ lower than expected. We do not know the reason for this; the period of Mermilliod \& Mayor is too accurate to allow such a large deviation at the orbital phase at which we observed VR $84(137.75 \pm 0.04)$.

The red spectra of H 209 were also cross correlated against each other to look for radial-velocity variations on time scales of hours. We found no variation within the errors $\left(1-2 \mathrm{~km} \mathrm{~s}^{-1}\right)$.

As stellar rotation and magnetic activity are closely linked, we also determine projected rotational velocities. Rotational velocities were measured from cross correlations with synthetic spectra (Kurucz 1979). Synthetic spectra of $T_{\text {eff }}=5500 \mathrm{~K}$, gravity $\log g=3.0$, turbulent velocity $v_{\text {turb }}=2 \mathrm{kms}^{-1}$ and $v_{\text {rot }} \sin i$ varying between 0 and $19 \mathrm{~km} \mathrm{~s}^{-1}$ in steps of $1 \mathrm{~km} \mathrm{~s}^{-1}$ were calculated 
between 4970 and $5040 \AA$. The object and synthetic spectra were correlated against the $v_{\text {rot }} \sin i=0 \mathrm{~km} \mathrm{~s}^{-1}$ spectrum; the peak of the cross correlation functions are fitted with Gaussian profiles. With the results we make a calibration curve that gives the width of the cross correlation peak as function of rotational velocity. The rotation for the objects then follows from comparison of the measured widths of their peaks with this calibration curve (see Table 1). The widths of the correlation peaks from $\mathrm{H} 3$ and H 11 are smaller than the smallest widths in the calibration curve. We note that the velocity thus derived is sensitive to the spectral type of the template. For example, correlation with a template of $T_{\text {eff }}=5000 \mathrm{~K}$ (same $\log g$ ) gives rotation velocities that are $\sim 1 \mathrm{~km} \mathrm{~s}^{-1}$ lower.

For the rapid rotator $\mathrm{H} 214 v_{\text {rot }} \sin i$ was determined with the method of Fourier-Bessel transformation (see Piters et al. 1996; Groot et al. 1996) that we also applied to the spectrum of the rapid rotator S 1113 in M 67 (van den Berg et al. 1999). We used three Fe I lines at $6400.15,6408.03$ and $6411.54 \AA$ to find an average $v_{\text {rot }} \sin i$ of $64 \mathrm{~km} \mathrm{~s}^{-1}$, a typical value for a star of this spectral type (Groot et al. 1996). The error in the cleanest line is $1.6 \mathrm{~km} \mathrm{~s}^{-1}$, in the most noisy line $10 \mathrm{~km} \mathrm{~s}^{-1}$. We determined no rotational velocity for the blue straggler H 209 for which Schönberner \& Napiwotzki (1994) determined a value of $v_{\text {rot }} \sin i$ of $50 \mathrm{~km} \mathrm{~s}^{-1}$ from highresolution spectra.

\subsection{Lithium $6708 \AA$}

As the element lithium is easily destroyed at temperatures higher than $\sim 2 \times 10^{6} \mathrm{~K}$, it can be used to discriminate between first-ascent giants, with outer layers that are not yet completely mixed to deeper, hotter layers by the development of a convective envelope, and clump stars. Discrimination between the two evolutionary phases can be difficult based on the position of a star in the colourmagnitude diagram alone as the position of the clump is close to the track of the first-ascent giants. Verbunt \& Phinney (1995) suggested that the orbital eccentricity of binaries containing a giant can be of help: the present radius of a clump giant is smaller than its radius at the tip of the giant branch; as the time scale for tidal circularization $\tau_{\text {circ }}$ is very sensitive to the ratio of the radius of the star with the dissipative tides $R$ to the semi-major axis $a$ $\left(\tau_{\text {circ }}^{-1} \sim(R / a)^{8}\right)$, clump stars can have circularized orbits that are too wide to be circularized by first-ascent giants with the same radius. Based on the circular orbits of VR 84 and VR 100 Verbunt \& Phinney therefore concluded that the giants in these binaries must be clump stars. We can test this prediction now by looking at the presence of the Lithium $6707.8 \AA$ line: whereas it is absent in VR 100 as expected from the prediction of Verbunt \& Phinney the line is visible in the spectrum of VR 84 which implies that the giant in VR 84 is a first-ascent instead of a clump giant (see Fig. 7).

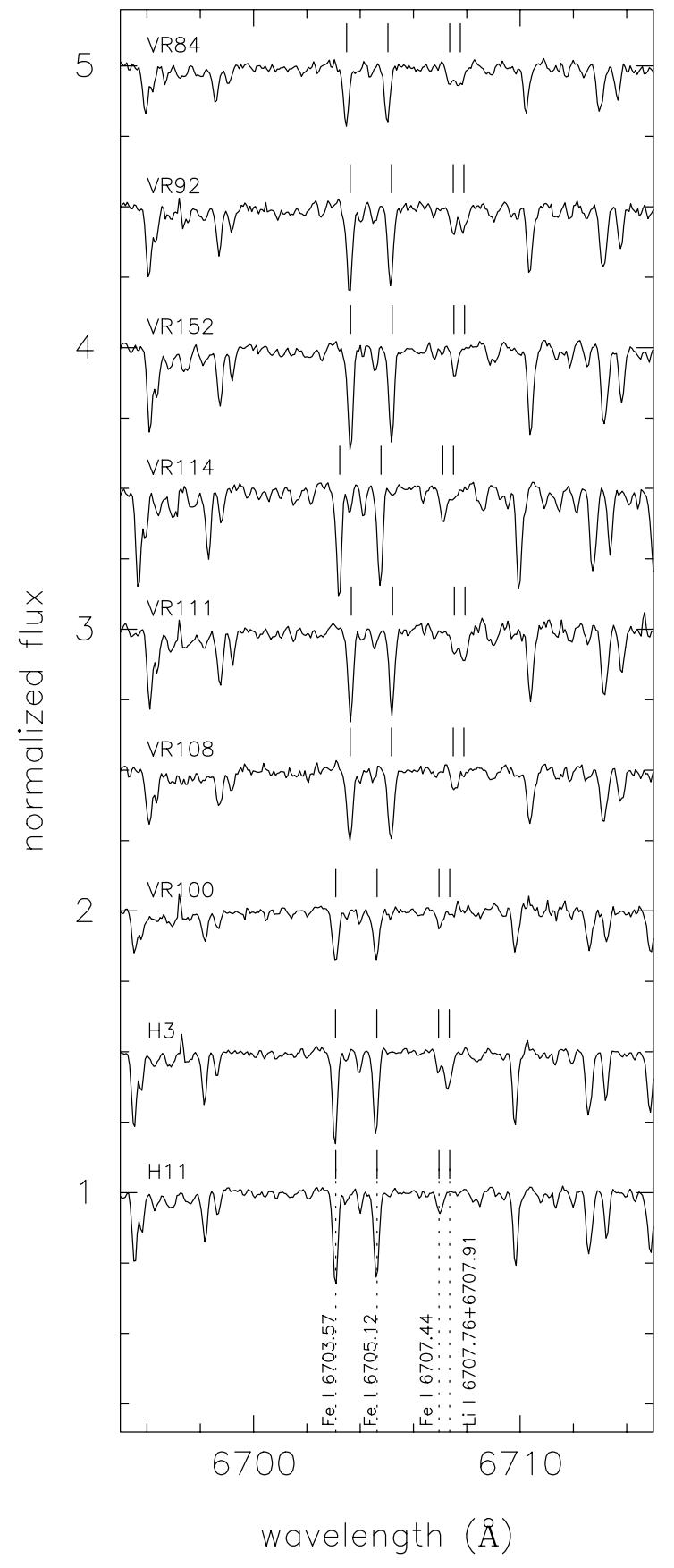

Fig. 7. The region around the Li I $6707.76 \AA$, $6707.91 \AA$ doublet. The expected positions of the lines are derived from the radial-velocity measurements.

How can it be that the orbit of VR 84 is circular? One possibility is that the decomposition of VR 84 into the two binary components (Mermilliod \& Mayor 1989) underestimates the radius of the giant. However, according to the calculations by Verbunt \& Phinney the radius has to increase to $\sim 16 R_{\odot}$ which cannot be accounted for by varying the reddening or distance modulus within the ranges that are found for NGC $6940(0.2<E(B-V)<0.3$, $(m-M)_{0}=9.7$; Larsson-Leander 1964) or by making the giant cooler (if $T_{\text {eff }}=4420 \mathrm{~K}$ then $R \approx 15 R_{\odot}$ according to the tables of Schmidt-Kaler; however this gives an 
observed $B-V=1.42$ which is redder than all the giants in NGC 6940). Alternatively, the orbit was not circularized by the present giant but during the giant-phase of its companion that in that case would be a white dwarf now. To account for the observed blue colours of VR 84 the companion white dwarf must be young and bright. The probability that we see both stars in VR 84 in relatively short-lived evolutionary phases is small. A whitedwarf companion is not excluded by the spectroscopic orbit that places a lower limit on the mass of the companion of $0.65 M_{\odot}$.

As an aside, we report on the observation of the Li I line in the two comparison giants H 3 and H11. The red clump of core-helium-burning stars in NGC 752 has attracted some attention: Mermilliod et al. (1998) pointed out four stars, including $\mathrm{H} 3$ and H11, below and to the blue of the main clump whose position is not reproduced by theoretical isochrones. Knowledge about the evolutionary phase of $\mathrm{H} 3$ and $\mathrm{H} 11$ could provide more insight into the situation. Daniel et al. (1994) predicted that H11 would be depleted of lithium, whereas lithium would be present in $\mathrm{H} 3$, that lies more towards the first ascent track; no lithium was found in the other two stars below the main clump (Pilachowski et al. 1988).

These expectations are confirmed by our observations (see Fig. 7) that show the Li I doublet in H3, while it is absent in H11. Thus the three bluest stars under the clump likely form a real extension to the clump. Girardi et al. (2000) suggested that the spread seen in the clump of NGC 752 results from the simultaneous presence of clump stars that have undergone the helium flash (the main clump) and of more massive stars that start helium burning more quiescently (at fainter magnitudes); a similar spread near the clump has been observed in the open cluster NGC 7789 that has about the same age as NGC 752.

\section{Discussion and conclusions}

In this paper we report on photometric and spectroscopic observations of four X-ray sources in the old open clusters NGC 752 and NGC 6940, for which the X-rays are unexplained. We conclude that our observations have not provided an answer to the question why these stars emit X-rays. Could it be that the optical identifications of the X-ray sources are wrong? Belloni \& Verbunt (1996) show that the probability for a chance identification in the field of NGC 752 is only 1\%. In NGC 6940, the suggested optical counterparts are so bright (between 11.5 and 10.4) that the probability for a misidentification is small. We conclude that the stars that we have studied are indeed the optical counterparts to the X-ray sources.

The light curves of the blue straggler H 209 in NGC 752 do not confirm earlier reports of variability (Hrivnak 1977); nor is an indication for variability seen in the spectra. Two other blue stragglers in open clusters (in M 67 and in IC 4651) were detected in X-rays. The former turned out to contain a 1.07-day period binary that could have a late-type magnetically active component (van den Berg et al. 2001a). No evidence for a similar system is seen in H 209. The source of the X-ray emission in H 209 and its blue-straggler nature remain a mystery.

The giants VR 108, VR 111 and VR 114 in NGC 6940 are of later spectral type and therefore magnetic activity in their convective outer layers could in principle be a cause for X-ray emission; though strong activity would be surprising as VR 111 is a wide binary and VR 108 shows no indication for binarity. The Ca II H\&K emission fluxes that we measure in VR 111 and VR 114 are indeed lower than is typical for magnetically active stars of their luminosity class; the asymmetric emission cores in the Ca II $\mathrm{H} \& \mathrm{~K}$ lines could be a signature of mass outflow as suggested by Dupree et al. (1999), who found similar emission cores in giants of various masses and temperatures. Also, the stars are not found to be rapid rotators. Therefore the $\mathrm{X}$-rays are probably not a result of enhanced magnetic activity in the giants themselves. Similar optical counterparts to X-ray sources - with long orbital periods or no indication for binarity - were identified in the older cluster M 67 (S 1237, S 760, S 775, S 1270). Apparently another mechanism than activity gives rise to the X-rays of the giants. Alternatively, these stars are triples that contain a hitherto undetected close binary, e.g. because the close binary is relatively faint or because its spectral signature is masked by rapid rotation of the component stars. Activity could be enhanced in VR 108; interestingly, the rotation velocity that we measure is somewhat higher than in the other giants. The reason for an enhanced activity level is not clear.

We confirm that VR 114 is located at about the same distance as the cluster. The information that we add here is that, like its proper motion, its radial velocity is not compatible with the cluster average. More radial-velocity measurements are needed to establish its binary nature.

We find that the short-period binary H 313 in NGC 752 is a photometric variable, and that its light curve suggests variability on the orbital period. If the latter can be confirmed, the rotation of one or both of the stars is likely tidally locked to the orbit. The X-rays - and the photometric variability - can thus be explained as coming from an active, spotted, rapidly rotating star, as suggested by Belloni \& Verbunt (1996).

Acknowledgements. The authors wish to thank Gertie Geertsema and Marco Kouwenhoven for their help with the observations, and Keivan Stassun for providing the routines to perform the light curve and photometric variability analysis. We also want to thank David Latham for the information on the binarity of $\mathrm{H} 209$; the measurements were taken as part of a larger study to monitor the radial velocities of blue stragglers in open clusters carried out by D. Latham and A. Milone. This research made extensive use of the WEBDA Open Cluster Database developed and maintained by J.-C. Mermilliod. M.vdB. is supported by the Netherlands Organization for Scientific Research (NWO). 


\section{References}

Belloni, T. 1997, in Cool stars in clusters and associations: magnetic activity and age indicators, ed. G. Micela, R. Pallavicini, \& S. Sciortino, 68, 993

Belloni, T., \& Tagliaferri, G. 1997, A\&A, 326, 608

Belloni, T., \& Verbunt, F. 1996, A\&A, 305, 806

Belloni, T., Verbunt, F., \& Mathieu, R. D. 1998, A\&A, 339, 431

Carraro, G., \& Chiosi, C. 1994, A\&A, 287, 761

Daniel, S. A., Latham, D. W., Mathieu, R. D., \& Twarog, B. A. 1994, PASP, 106, 281

Dinescu, D. I., Demarque, P., Guenther, D. B., \& Pinsonneault, M. H. 1995, AJ, 109, 2090

Dupree, A. K., Whitney, B. A., \& Pasquini, L. 1999, ApJ, 520, 751

Fernández-Figueroa, M. J., Montes, D., de Castro, E., \& Cornide, M. 1994, ApJS, 90, 433

Garrison, R. F. 1972, ApJ, 177, 653

Girardi, L., Mermilliod, J.-C., \& Carraro, G. 2000, A\&A, 354, 892

Groot, P. J., Piters, A. J. M., \& van Paradijs, J. 1996, A\&AS, 118,545

Heinemann, K. 1926, Astr. Nach., 227, 193

Honeycutt, R. K. 1992, PASP, 104, 435

Hrivnak, B. J. 1977, IBVS, 1293

Janes, K. A., \& Phelps, R. L. 1994, AJ, 108, 1773

King, D. L. 1985, ING Technical Note, 31

Kurucz, R. L. 1979, ApJS, 40, 1
Larsson-Leander, G. 1964, ApJ, 140, 144

Mallik, S. V. 1994, A\&AS, 103, 279

Malyuto, V., \& Schmidt-Kaler, T. 1997, A\&A, 325, 693

Massey, P., Strobel, K., Barnos, J. V., \& Anderson, E. 1988, ApJ, 328, 315

Mermilliod, J.-C., Mathieu, R. D., Latham, D. W., \& Mayor, M. 1998, A\&A, 339, 423

Mermilliod, J.-C., \& Mayor, M. 1989, A\&A, 219, 125

Milone, E. F., Stagg, C. R., Sugars, B. A., et al. 1995, AJ, 109, 359

Pilachowski, C. A., Saha, A., \& Hobbs, L. M. 1988, PASP, 100, 474

Piters, A. J. M., Groot, P. J., \& van Paradijs, J. 1996, A\&AS, 118,529

Rebeirot, E. 1970, A\&A, 4, 404

Sanders, W. L. 1972, A\&A, 16, 58

Scargle, J. D. 1982, ApJ, 263, 835

Schönberner, D., \& Napiwotzki, R. 1994, A\&A, 282, 106

van den Berg, M., Orosz, J., Verbunt, F., \& Stassun, K. 2001a, A\&A, 375, 375

van den Berg, M., Stassun, K., Verbunt, F., \& Mathieu, R. D. 2001b, A\&A, submitted

van den Berg, M., Verbunt, F., \& Mathieu, R. D. 1999, A\&A, 347,866

van den Bergh, S., \& McClure, R. D. 1980, A\&A, 88, 360

Vasilevskis, S., \& Rach, R. A. 1957, AJ, 62, 157

Verbunt, F., \& Phinney, E. S. 1995, A\&A, 296, 709

Walker, M. F. 1958, ApJ, 128, 562 